\title{
The Superfluid and Conformal Phase Transitions of Two-Color QCD
}

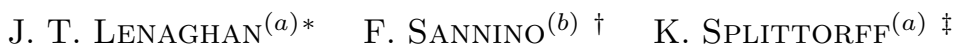 \\ (a) The Niels Bohr Institute \& ${ }^{(b)}$ NORDITA \\ Blegdamsvej 17, DK-2100 Copenhagen Ø, Denmark
}

(July 2001)

\begin{abstract}
The phase structure of two-color QCD is examined as a function of the chemical potential and the number of light quark flavors. We consider effective Lagrangians for two-color QCD containing the Goldstone excitations, spin-one particles and negative intrinsic parity terms. We discuss the possibility of a conformal phase transition and the enhancement of the global symmetries as the number of flavors is increased. The effects of a quark chemical potential on the spin-one particles and on the negative intrinsic parity terms are analyzed. It is shown that the phase diagram that is predicted by the linearly realized effective Lagrangian at tree-level matches exactly that predicted by chiral perturbation theory.
\end{abstract}

\section{INTRODUCTION}

Quantum chromodynamics (QCD) at large quark chemical potential has attracted a great deal of interest in recent years 仼. Since single gluon exchange between two quarks is attractive in the color-antitriplet channel [2,3], quark matter is expected to behave as a color superconductor for a sufficiently large quark chemical potential. Possible phenomenological applications include the description of quark stars, neutron star interiors and the physics near the core of collapsing stars [1. [. diagram from first principles as a function of temperature, chemical potential and the number of light flavors. While much has been learned about the phase structure of QCD at nonzero temperature through a combination of perturbation theory and lattice simulations, the phase structure at nonzero chemical potential and for large numbers of flavors has been less extensively explored [6]. The phase structure as the number of light flavors is increased is expected to be quite rich. For example, in Ref. [7], the effects of chiral symmetry breaking were found to be dramatically reduced as the number of flavors was increased from zero to four. At asymptotically large quark chemical potentials, $\mu \gg \Lambda_{\mathrm{QCD}}$, perturbation theory is valid and one is able to perform controlled calculations. For small to intermediate chemical potentials, however, one must rely either on effective theories or perform lattice simulations. Standard importance sampling methods employed in lattice simulations fail, however, at nonzero chemical potential for $N_{c}=3$ since the fermionic determinant is complex.

For $N_{c}=2$, the situation is very different since the quarks are in a pseudoreal representation of the gauge group. The first difference is that the fermionic determinant in the path integral is real (thought not necessarily positive) and so lattice simulations can be performed at nonzero baryon and isospin chemical potential for an even number of quark flavors 8 19]. Secondly, $N_{f}^{2}-N_{f}$ of the Goldstone excitations are diquarks which carry nonzero baryon charge. As shown in Refs. [20,21, this has the advantage that chiral perturbation theory [22] is valid at the critical chemical potential as opposed to the case of $N_{c}=3$ for which the critical chemical potential lies well above the scale at which chiral perturbation theory becomes invalid. Also, the formation of a diquark condensate in the $N_{c}=2$ theory does not break gauge invariance and so exhibits superfluidity at large chemical potentials, unlike the case of $N_{c}=3$ which exhibits superconductivity.

\footnotetext{
*Electronic address: lenaghan@alf.nbi.dk

${ }^{\dagger}$ Electronic address: sannino@alf.nbi.dk

${ }^{\ddagger}$ Electronic address: split@alf.nbi.dk
} 
There also has been much progress in understanding the phase structure of supersymmetric theories as the number of massless fermions is varied [23]. While much less in known for nonsupersymmetric theories, the infrared behavior of such theories should change dramatically as the number of massless fermions is increased. In particular, for $N_{f}>11$, the one-loop beta function for $N_{c}=2$ QCD becomes positive and the theory loses asymptotic freedom. In this non-Abelian QED-like phase, the theory is not expected to be confining or to exhibit chiral symmetry breaking. Just below $N_{f}=11$, a perturbative infrared stable fixed point develops. In this phase, the trace of the energy momentum tensor vanishes and the theory is a non-Abelian conformal field theory. Just before the onset of the conformal phase, it has been argued that an enhanced global symmetry can emerge involving the massive spectrum of the theory 24,25. This new dynamical symmetry may arise when the number of light flavors is near a critical number of flavors (about $N_{F}=8$ [24 27]). Above this value, the theory is expected to enter the conformal regime. This enhanced symmetry group may be important, for example, when coupling a two-color strongly interacting theory to the electroweak symmetry breaking sector of the standard model 24]. The hadronic spectrum close to this point can be very different than for a smaller number of flavors. For example, this enhanced global symmetry can lead to degenerate masses for the vector spectrum even in the presence of chiral symmetry breaking. Additionally, when approaching the conformal point all of the massive states of the theory become light exponentially fast as the number of flavors reaches the critical value.

In this work, we study the phase structure of $N_{c}=2 \mathrm{QCD}$ as a function of chemical potential and the number of light flavors. We begin by reviewing the most general effective Lagrangian for $N_{c}=2$ describing the Goldstone bosons and spin-one states and their interactions. This Lagrangian was first constructed in Refs. [24] and 28] within the context of extended technicolor theories. We first discuss the nonlinear realization of chiral symmetry and later consider the linear effective Lagrangian. Before introducing a chemical potential, the possible phase structure of two-color QCD as the number of flavors is increased is discussed. The enhanced global symmetry mentioned above is identified at the level of the effective Lagrangian.

We stress that if we were to consider an approximate local flavor symmetry when introducing the spin-one particles, then no enhanced symmetry is allowed. An intriguing possibility is that for a small number of flavors relative to the number of colors it might be reasonable to include the vectors as almost gauge vectors of chiral symmetry, while for a large number of flavors the enhanced global symmetry may set in. Both limits severely constrain the effective Lagrangians. Lattice simulations are a very useful means of testing such conjectures.

An essential component for any effective Lagrangian for a strongly interacting theory is the set of intrinsic negative parity terms, i.e. those terms contracted by the fully antisymmetric tensor $\epsilon_{\eta \nu \rho \sigma}$. The Wess-Zumino term [29,30] is the time honored example of an $\epsilon$ term and is needed to saturate (in the Goldstone phase) the 't Hooft global non-Abelian anomaly constraints. When the underlying fermions are in a pseudoreal representation of the gauge group, the $\epsilon$ part of the effective Lagrangian involving the Goldstone bosons and the spin-one fields was explicitly constructed in Ref. [28]. We show that this sector of the theory couples to the baryon and isospin chemical potentials. These terms are also expected to be important when studying the solitonic sector of the theory.

Next, we study the effect of the chemical potential on the spin-one fields and we explicitly calculate the mass gaps. The results suggest that some of the vectors may condense and hence break rotational invariance. The value of the chemical potential at the onset of the vector condensation is predicted by assuming that vectors are sufficiently massive and that the vacuum alignment of the theory is determined by the Goldstone excitations.

We then turn to study the superfluid phase transition at nonzero chemical potential using a linear realization of the underlying chiral symmetry. The linear sigma model not only has the advantage that the calculations are relatively simple but also allows us to predict how the magnitude of the condensates changes with the baryon and isospin chemical potentials. The vacuum structure that is predicted at tree-level is identical to that of chiral perturbation theory [31]. The dispersion relations of the pseudo-Goldstone bosons are calculated as well as the dependence of the condensates on the baryon and isospin chemical potentials.

This paper is organized as follows. In Sec. III, the nonlinearly realized effective Lagrangian is 
reviewed. In Sec. III we briefly comment on the $N_{f}$ phase diagram at zero chemical potential and review the possibility [24,25,28] that novel phenomena like parity doubling can emerge near the conformal phase transition. The effects of a nonzero baryon chemical potential are illustrated in Secs. IV, V, and VI. First, we study the effects of the chemical potential on the $\epsilon$ terms and then we consider the dispersion relations of the spin-one fields in the presence of a chemical potential. We then turn to the linearly realized theory. In Sec. VII, the linear effective Lagrangian is reviewed. The phase diagram and dispersion relations at nonzero baryon and isospin chemical potentials are studied and the results are contrasted with those of chiral perturbation theory. We conclude in Sec. VIII. Our conventions are summarized in the Appendix.

\section{THE TWO-COLOR NONLINEAR EFFECTIVE LAGRANGIAN}

The simplest example of a gauge theory with fermions in a pseudoreal representation is $N_{c}=2$ QCD with the fermions in the fundamental representation. The quantum global symmetry for $N_{f}$ matter fields is $S U\left(2 N_{f}\right)$ which contains $S U_{L}\left(N_{f}\right) \times S U_{R}\left(N_{f}\right)$. Using the Wess and Bagger spinorial conventions [32], the underlying Lagrangian is

$$
\mathcal{L}_{N_{c}=2}=-\frac{1}{4 g^{2}} \vec{G}_{\mu \nu} \cdot \vec{G}^{\mu \nu}+i \bar{Q} \bar{\sigma}^{\nu}\left[\partial_{\nu}-i \overrightarrow{G_{\nu}} \cdot \frac{\vec{\tau}}{2}\right] Q-\frac{1}{2} m_{q} Q^{T} \tau_{2} E Q+\text { h.c. } .
$$

$G_{\mu \nu}^{a}$ and $G_{\nu}^{a}$ with $a=1,2,3$ are the gluon field strength and field respectively, while the $\tau^{a}$ are the Pauli matrices for the $S U_{c}(2)$ group. $Q_{\alpha}^{c, I}$ is a two-spinor fermion field in the fundamental representation of color with $c=1,2$ and $I=1, \cdots, 2 N_{f}$,

$$
Q=\left(\begin{array}{c}
q_{L} \\
i \sigma_{2} \tau_{2} q_{R}^{*}
\end{array}\right)
$$

In the massless limit $\left(m_{q}=0\right)$, the classical global symmetry is $U\left(2 N_{f}\right)$ which is then broken by the Adler-Bell-Jackiw anomaly to $S U\left(2 N_{f}\right)$. The mass term explicitly breaks the $S U\left(2 N_{f}\right)$ symmetry to $S p\left(2 N_{f}\right)$. The $2 N_{f} \times 2 N_{f}$ matrix $E$ is

$$
E=\left(\begin{array}{cc}
0 & 1 \\
-1 & 0
\end{array}\right)
$$

For a sufficiently small number of flavors, one expects the theory to confine and to dynamically generate a condensate which spontaneously breaks the global symmetry group for zero quark masses. For three-color QCD with quarks in the fundamental representation, this condensate is the usual quark-antiquark condensate; however, on account of the enlarged $S U\left(2 N_{f}\right)$ global symmetry in two-color QCD, any quark-antiquark condensate can be continuously rotated into a quark-quark condensate and the only discernible condensate is an admixture of these two condensates. The subgroup down to which the $S U\left(2 N_{f}\right)$ symmetry is broken is usually taken to be the maximal diagonal subgroup $S p\left(2 N_{f}\right)$ [33], a choice which is consistent with a new criterion presented in Ref. [34].

We now turn to the construction of the low energy effective Lagrangian. We divide the hermitian generators, $\left\{T^{a}\right\}$, of $S U\left(2 N_{f}\right)$, normalized according to $\operatorname{Tr}\left[T^{a} T^{b}\right]=\delta^{a b} / 2$, into two classes: the generators of $S p\left(2 N_{f}\right)$ which we denote by $\left\{S^{a}\right\}$ with $a=1, \ldots, 2 N_{f}^{2}+N_{f}$, and the remaining generators of $S U\left(2 N_{f}\right)$ which we denote by $\left\{X^{i}\right\}$ with $i=1, \ldots, 2 N_{f}^{2}-N_{f}-1$. Note that the latter set parameterizes the quotient space $S U\left(2 N_{f}\right) / S p\left(2 N_{f}\right)$. An explicit realization of the generators is provided in the Appendix. This breaking pattern gives $2 N_{f}^{2}-N_{f}-1$ Goldstone bosons which are encoded in the $2 N_{f} \times 2 N_{f}$ antisymmetric matrix

$$
U=e^{i \frac{\Pi^{j} X^{j}}{v}} E .
$$


$U$ transforms linearly under a chiral rotation as

$$
U \rightarrow u U u^{T}
$$

with $u \in S U\left(2 N_{f}\right)$. The nonlinear realization constraint, $U U^{\dagger}=1$, is automatically satisfied.

The generators of the $S p\left(2 N_{f}\right)$ satisfy the relation

$$
S^{T} E+E S=0
$$

while the $X^{i}$ generators obey

$$
X^{T} E-E X=0
$$

Using this last relation we can easily demonstrate that $U^{T}=-U$. For simplicity, we also require that

$$
\operatorname{Pf} U=1
$$

in order to avoid discussing the explicit realization of the underlying Adler-Bell-Jackiw axial anomaly at the effective Lagrangian level 27.

\section{A. The Spin-One Fields}

We next introduce the coupling between the Goldstone excitations and a vector field. While there are many different ways to introduce vector fields at the level of the effective Lagrangian (the hidden local gauge symmetry of Ref. [35], for example), they are all equivalent at tree-level. We consider the vector field

$$
A_{\nu}=A_{\nu}^{a} T^{a}
$$

which we take to transform under a $S U\left(2 N_{f}\right)$ rotation as

$$
A_{\nu} \rightarrow u A_{\nu} u^{\dagger}-i\left(\partial_{\nu} u\right) u^{\dagger}
$$

It is useful to formally define a chiral covariant derivative

$$
D_{\nu} U=\partial_{\nu} U-i A_{\nu} U-i U A_{\nu}^{T} .
$$

The most general two-derivative term which preserves local chiral symmetry is

$$
\operatorname{Tr}\left[D_{\nu} U D^{\nu} U^{\dagger}\right] \text {. }
$$

Although we have introduced the vector fields formally as vectors associated with a local chiral gauge theory, the effective Lagrangian must respect the global $S U\left(2 N_{f}\right)$ transformations and is given by

$$
\begin{aligned}
\mathcal{L}_{e f f} & =v^{2} \operatorname{Tr}\left[D_{\nu} U D^{\nu} U^{\dagger}\right]+m_{V}^{2} \operatorname{Tr}\left[A_{\nu} A^{\nu}\right]+h v^{2} \operatorname{Tr}\left[A_{\nu} U A^{T \nu} U^{\dagger}\right] \\
& +i s v^{2} \operatorname{Tr}\left[A_{\nu} U D^{\nu} U^{\dagger}\right]+v^{2} m_{\pi}^{2} \operatorname{Tr}\left[\mathcal{M} U+\mathcal{M}^{\dagger} U^{\dagger}\right]
\end{aligned}
$$

We counted the vector fields as derivatives and added a democratic quark mass matrix

$$
\mathcal{M} \equiv\left(\begin{array}{cc}
0 & -1 \\
1 & 0
\end{array}\right)
$$

The parameters $m_{V}, s, h$ are real constants which effectively measure the departure from local chiral symmetry. The degenerate masses of the pseudo-Goldstone exicitations are denoted by $m_{\pi}$. 
Equation (13) is the most general two-derivative effective Lagrangian compatible with the global symmetries of $N_{c}=2$ QCD [24,28].

For completeness, we augment the effective Lagrangian with the simplest possible kinetic term for the vectors:

$$
\mathcal{L}_{k i n}=-\frac{1}{2 g^{2}} \operatorname{Tr}\left[F_{\rho \nu} F^{\rho \nu}\right]
$$

where

$$
F^{\rho \nu}=\partial^{\rho} A^{\nu}-\partial^{\nu} A^{\rho}-i\left[A^{\rho}, A^{\nu}\right]
$$

The vector kinetic piece arises as a fourth order term in the derivative counting and $g$ is a dimensionless coupling constant. The tree-level masses of the vectors can also be calculated and are given by

$$
\begin{aligned}
& M_{S}^{2}=g^{2}\left(m_{V}^{2}-h v^{2}\right) \\
& M_{X}^{2}=g^{2}\left(m_{V}^{2}+v^{2}(h+4-2 s)\right),
\end{aligned}
$$

where we have split the vectors into those associated with the $\left\{S^{a}\right\}$ generators and those associated with the $\left\{X^{a}\right\}$ generators. Note that, in general, there is a nonzero mass splitting between these two sets.

\section{B. The $\epsilon$ terms for $S U\left(2 N_{f}\right)$}

Next, we consider the complete set of 4-derivative terms which have negative intrinsic parity and contain spin-one and spin-zero fields. As mentioned in the introduction, these terms contain the Lorentz antisymmetric tensor $\epsilon_{\eta \nu \rho \sigma}$ and the canonical example is the Wess-Zumino action. These terms are necessary at the effective Lagrangian level since they account for the 't Hooft global anomaly constraints in the Goldstone phase. Additionally, they are important when quantizing the solitonic sector of the theory. They can be compactly written using the language of algebra-valued differential forms:

$$
\alpha=(d U) U^{-1}=\left(\partial_{\nu} U\right) U^{-1} d x^{\nu}
$$

Since the fermions are in a pseudoreal representation of the gauge group, it is sufficient to define only one type of differential form, $\alpha$, since now the other possible form $\beta=U^{-1} d U=\alpha^{T}$ is not independent 28]. The Wess-Zumino term is

$$
\Gamma_{W Z}[U]=C \int_{M^{5}} \operatorname{Tr}\left[\alpha^{5}\right] .
$$

The dimension of the spacetime must be increased by one spatial direction in order to make the action local. Hence, the integral in eq. (18) must be performed over a five-dimensional manifold whose boundary $\left(M^{4}\right)$ is the ordinary four-dimensional Minkowski space. The coefficient $C$ can be fixed, in general, by matching the anomalous variation of the currents associated with non-Abelian anomalies at the effective Lagrangian level. For the case at hand, the constant $C$ is fixed to be

$$
C=-i \frac{N_{c}}{240 \pi^{2}}
$$

with $N_{c}=2$ [28]. We note that the coefficient is similar to the case $N_{c}=3$ since $S U\left(2 N_{f}\right) \supset$ $S U_{L}\left(N_{f}\right) \times S U_{R}\left(N_{f}\right)$.

Since we are considering a theory which contains vectors and only global chiral symmetry, eq. (18) needs to be generalized. This has been done in Ref. 28. In order to generate all allowed terms, the 
authors of Ref. 28 first formally gauged the Wess-Zumino action following a standard procedure developed in Refs. 30,36, 37]. This procedure automatically provides most of the desired terms and local chiral invariance relates the coefficients of the new $\epsilon$ terms to the Wess-Zumino coefficient. The effective Lagrangian was then generalized to be only globally invariant under chiral rotations, and as a result, all the terms have different coefficients. Remarkably, the gauging procedure generates all but one term allowed by global invariance.

The most general 4-derivative $\epsilon$ Lagrangian respecting global chiral rotations is

$$
\begin{aligned}
\Gamma_{W Z}[U, A]= & \Gamma_{W Z}[U]+i 10 C_{1} \int_{M^{4}} \operatorname{Tr}\left[A \alpha^{3}\right] \\
& -10 C_{2} \int_{M^{4}} \operatorname{Tr}[(d A A+A d A) \alpha] \\
& -5 C_{3} \int_{M^{4}} \operatorname{Tr}\left[d A d U A^{T} U^{-1}-d A^{T} d U^{-1} A U\right] \\
& -5 C_{4} \int_{M^{4}} \operatorname{Tr}\left[U A^{T} U^{-1}\left(A \alpha^{2}+\alpha^{2} A\right)\right] \\
& +5 C_{5} \int_{M^{4}} \operatorname{Tr}\left[(A \alpha)^{2}\right]+i 10 C_{6} \int_{M^{4}} \operatorname{Tr}\left[A^{3} \alpha\right] \\
& +i 10 C_{7} \int_{M^{4}} \operatorname{Tr}\left[(d A A+A d A) U A^{T} U^{-1}\right] \\
& -i 10 C_{8} \int_{M^{4}} \operatorname{Tr}\left[A \alpha A U A^{T} U^{-1}\right]+10 C_{9} \int_{M^{4}} \operatorname{Tr}\left[A^{3} U A^{T} U^{-1}\right] \\
& +\frac{5}{2} C_{10} \int_{M^{4}} \operatorname{Tr}\left[\left(A U A^{T} U^{-1}\right)^{2}\right] \\
& +i C_{11} \int_{M^{4}} \operatorname{Tr}\left[A^{2}\left(\alpha U A^{T} U^{-1}-U A^{T} U^{-1} \alpha\right)\right]
\end{aligned}
$$

where $C_{i}$ are imaginary coefficients and $A=A^{\nu} d x_{\nu}$ [28]. It is important to stress that when imposing local chiral symmetry all of the coefficients are given by eq. (19) except for $C_{11}$ which is zerot. Using $\mathrm{C}$ and $\mathrm{CP}$ invariance, one can show that there are no other negative intrinsic odd parity terms at this order. Aside from the standard four-derivative terms involving the Goldstone fields, we are now endowed with a rather complete and general effective Lagrangian. The action is

$$
S_{e f f}=\int d^{4} x\left(\mathcal{L}_{e f f}+\mathcal{L}_{k i n}\right)+\Gamma_{W Z}[U, A]
$$

\section{THE PHASE STRUCTURE ALONG THE $N_{F}$-AXIS}

As mentioned in the Introduction, the infrared behavior of gauge theories changes dramatically as the number of light fermion flavors is varied [23. In this section, we review two possibilities that should be directly accessible in lattice simulations of two-color QCD. Predictions resulting from analytic calculations are at the moment only possible for supersymmetric theories; however, the behavior of the beta function for two-color QCD does offer some guidance. We focus here on zero chemical potential, but in later sections will briefly discuss the large- $N_{f}$ behavior of two-color QCD at nonzero chemical potential.

\footnotetext{
${ }^{1} \mathrm{NB}$ : We have changed the normalization of the coefficients with respect to Ref. 28]
} 


\section{A. The Conformal Phase Transition}

For $N_{f}>11$, the one-loop beta function of two-color QCD changes sign and the theory loses asymptotic freedom [39,40]. The resulting infrared free theory is now in a non-Abelian QED-like phase in which neither confinement nor chiral symmetry breaking is expected. For values of $N_{f}$ near but below 11, the beta function develops a perturbative infrared stable fixed point at which the trace of the energy momentum tensor vanishes exactly and the theory is a non-Abelian conformal field theory. In this phase, the coupling constant is small on account of the large number of flavors and so we do not expect any of the global symmetries to break. However, as the number of flavors is decreased, the fixed point becomes nonperturbative and the coupling constant increases to a critical value at which chiral symmetry is spontaneously broken. A dynamical scale is generated and conformal symmetry is lost. The generation of this scale defines the critical number of massless flavors, i.e. the minimum number of flavors for which the gauge theory is still conformal and chiral symmetry is still intact. Below this critical number of flavors, the theory is expected to confine and the low energy spectrum is hadronic. This discussion assumes that the conformal and chiral phase transitions coincide, but whether or not this is true is still controversial. We will assume here, as corroborated by lattice simulations for $N_{c}=3$ [7], that there is in fact a single conformal/chiral phase transition. Figure 1 summarizes the possible phase structure for two-color QCD as a function of the number of light flavors.

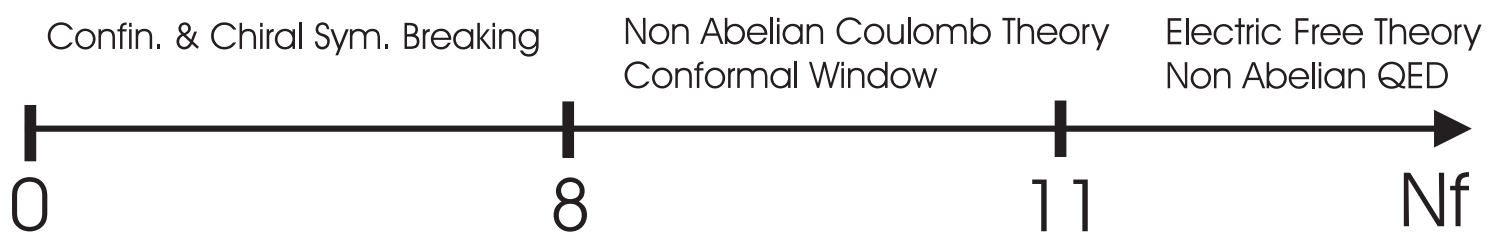

FIG. 1. The possible phase structure of $N_{c}=2 \mathrm{QCD}$ as function of the number of light quark flavors.

\section{B. The Enhanced Global Symmetry Scenario}

When the number of flavors is just below the critical value, the theory still exhibits chiral symmetry breaking but it is possible that the vector spectrum changes quite significantly. In Refs. 24,28], it was suggested that a new global symmetry may be dynamically generated. This symmetry acts on the massive spectrum of the theory and it is related to the modification of the second Weinberg spectral function sum rule near the critical number of flavors [25]. Indeed, there are examples of supersymmetric theories with enhanced global symmetry groups [38].

From eq. (13), one sees that the global symmetry group becomes $S p\left(2 N_{f}\right) \times\left[S U\left(2 N_{f}\right)\right]$ for

$$
s=4, \quad h=2,
$$

and the mass splitting between the vectors is zero:

$$
M_{S}^{2}=g^{2}\left(m_{V}^{2}-2 v^{2}\right)=M_{X}^{2} .
$$

The extra $S U\left(2 N_{f}\right)$ symmetry group acts only on the vector field as

$$
A \rightarrow u A u^{\dagger}, \quad u \in\left[S U\left(2 N_{f}\right)\right],
$$

and the effective Lagrangian, eq. (13), reduces to:

$$
\mathcal{L}=v^{2} \operatorname{Tr}\left[\partial_{\nu} U \partial^{\nu} U^{\dagger}\right]+M^{2} \operatorname{Tr}\left[A_{\nu} A^{\nu}\right],
$$

where $M^{2}=m_{V}^{2}-2 v^{2}$ [24.28]. Note that the Lagrangian also possesses an extra global $Z_{2}$ (i.e. $A \rightarrow z A$, with $z= \pm 1$ ) symmetry. If such an enhanced symmetry emerges, the vectors along the 
broken generators become mass degenerate with those along the orthogonal directions. For $N_{c}=3$ QCD, this corresponds to mass degenerate vector and axial particles even in the presence of chiral symmetry breaking.

From eq. (25), one finds that in the enhanced symmetry scenario, the interactions between the vectors and the Goldstone excitations only appear at the next order in the derivative counting scheme in the form of the double trace terms

$$
\mathcal{L}_{4}=+a_{1} \operatorname{Tr}\left[\partial_{\rho} U \partial^{\rho} U^{\dagger}\right] \operatorname{Tr}\left[A_{\nu} A^{\nu}\right]+a_{2} \operatorname{Tr}\left[\partial_{\rho} U \partial_{\nu} U^{\dagger}\right] \operatorname{Tr}\left[A^{\rho} A^{\nu}\right],
$$

where $a_{1}$ and $a_{2}$ are real coefficients. If the only extended symmetry group is the discrete $Z_{2}$ group, then the Lagrangian can include single trace terms of the form

$$
\operatorname{Tr}\left[\partial_{\rho} U \partial^{\rho} U^{\dagger} A_{\nu} A^{\nu}\right], \quad \operatorname{Tr}\left[A_{\rho} A^{\rho} U A_{\nu}^{T} A^{T \nu} U^{\dagger}\right] .
$$

The enhanced symmetry scenario imposes very stringent constraints on the possible form of the $\epsilon$ terms as well. If we require that the effective Lagrangian respects the full enhanced global symmetry, $S p\left(2 N_{f}\right) \times\left[S U\left(2 N_{f}\right)\right] \times Z_{2}$, then there are no vector axial $\epsilon$-terms. However, if only the discrete $Z_{2}$ symmetry is imposed, then the surviving terms are $C_{2}, C_{3}, C_{4}, C_{5}, C_{9}$ and $C_{10}$.

As supported by ordinary QCD phenomenology [36], we conjecture the following phase structure before entering the conformal phase:

- Approximate local chiral symmetry for small $N_{f}$

- Parity doubling and an extra global symmetry near the critical $N_{f}$.

For a fixed, nonzero chemical potential, the phase structure as the number of light flavors is increased should be even richer. For instance, when $N_{f}>11$, the theory is no longer asymptotically free and the low energy theory is simply the QCD Lagrangian. This regime is clearly not in the same universality class as the one in which the lowest excitations are Goldstone bosons. For $N_{f}$ smaller than but near the critical value, $N_{f} \simeq 8$, we approach the conformal phase. The hadronic mass scale of the theory vanishes exponentially fast [25 27] and all of the physical states whose masses are linked to the hadronic mass scale, i.e. all non-Goldstone excitations, become very light. When there is a large mass gap between the Goldstone excitations and the rest of the spectrum, the phase diagram for $N_{c}=2$ can be predicted using chiral perturbation theory [20 21]31. Near the conformal point, however, this approach is expected to break down since there is now a tower of light, non-Goldstone excitations. This tower of light states should suppress the formation of both the quark-antiquark and the diquark condensates by virtue of conformality. This behavior can be distinguished from the corrections to the mean-field analysis of the effective Lagrangian since the latter will not lead to a vanishing of either condensate. This has already been verified at next-to-leading order in Ref. [11]. We suggest then that by measuring diquark condensation at nonzero chemical potential lattice simulations may be able to single out important features of the conformal phase transition.

\section{NONZERO CHEMICAL POTENTIAL}

In this section, we review the procedure for introducing a chemical potential associated with a conserved charge into the effective theory. For simplicity, we consider only a baryon chemical potential, but the generalization to include an isospin chemical potential is straightforward. This procedure uniquely fixes the coefficients of the chemical potential terms in the effective Lagrangian and is equivalent to the approaches used in Refs. 20,21 to introduce a chemical potential via an auxiliary extended gauge symmetry.

At nonzero chemical potential, the microscopic Lagrangian has the form

$$
\mathcal{L}_{N_{c}=2}=-\frac{1}{4 g^{2}} \vec{G}_{\mu \nu} \cdot \vec{G}^{\mu \nu}+i \bar{Q} \bar{\sigma}^{\nu}\left[\partial_{\nu}-i \mu_{B} B \delta_{0 \nu}-i \overrightarrow{G_{\nu}} \cdot \frac{\vec{\tau}}{2}\right] Q-\frac{1}{2} m_{q} Q^{T} \tau_{2} E Q+\text { h.c. } .
$$


where the $2 N_{f} \times 2 N_{f}$ matrix

$$
B=\frac{1}{2}\left(\begin{array}{cc}
1 & 0 \\
0 & -1
\end{array}\right)
$$

is the baryon charge matrix for the quarks and the conjugate quarks?. One may check that when written in the basis of the usual $S U\left(2 N_{f}\right)$ spinors this term gives the usual coupling of the quarks to the chemical potential, $\mu_{B} \bar{\psi} \gamma_{0} \psi[21]$.

After defining $B_{\nu} \equiv \mu_{B} B \delta_{0 \nu}$, this Lagrangian is formally invariant under the following $S U\left(2 N_{f}\right)$ transformation

$$
\begin{aligned}
Q & \rightarrow u Q \\
B_{\nu} & \rightarrow u B_{\nu} u^{\dagger}-\frac{1}{\mu} u\left(\partial_{\nu} u^{\dagger}\right) \\
E & \rightarrow u^{*} E u^{\dagger},
\end{aligned}
$$

and $u \in S U\left(2 N_{f}\right)$. Implementing the previous transformations at the effective Lagrangian, the authors of Refs. [20,21,31] were able to uniquely determine the coupling of the chemical potential to the Goldstone bosons in the effective theory. The result is that the chemical potential enters into the effective Lagrangian in the form of a covariant derivative which we give schematically by

$$
\partial_{\nu} \rightarrow \partial_{\nu}-i B_{\nu}
$$

This same result can be arrived at by examining the conserved charges in the effective theory. The chemical potential is associated with the conserved charge of baryon number. Since the Lagrangian is invariant under a global $U(1)_{B}$ symmetry group, there is a conserved current, the zeroth component of which is the conserved charged associated with the baryon number. In the operator formalism, the grand canonical partition function is

$$
Z(\beta, \mu)=\operatorname{Tr} e^{-\beta(\hat{H}-\mu \hat{Q})} .
$$

Converting from the operator formalism to the path integral formalism, one finds that after integrating over the field momentum the introduction of a chemical potential, for a scalar degree of freedom, serves only to shift the time derivatives in the fashion:

$$
\frac{\partial^{2}}{\partial t^{2}} \rightarrow \frac{\partial^{2}}{\partial t^{2}}-2 \mu \frac{\partial}{\partial t}+\mu^{2}
$$

Note that this is equivalent to replacing the usual derivatives by the covariant derivative in eq. (31).

Before discussing the effects of a nonzero chemical potential on the vectors and the $\epsilon$-terms, it is instructive to review the predictions of the effective Lagrangian including only the pseudo-Goldstone excitations [20 21]. The effects of a nonzero baryon chemical potential are manifest even at the level of the chiral effective Lagrangian since $N_{f}\left(N_{f}-1\right)$ of the Goldstone modes are diquarks which have nonzero baryon charge. This is not the case for $N_{c}=3 \mathrm{QCD}$ since there is no coupling between the Goldstone modes and the baryon chemical potential in chiral perturbation theory. Another related, but salient, difference is that for $N_{c}=2$, the critical chemical potential at which the baryon density becomes nonzero is well within the range of validity of the effective theory.

Since a nonzero baryon chemical potential only preserves a $S p\left(N_{f}\right) \times S p\left(N_{f}\right)$ subgroup of the original $S U\left(2 N_{f}\right)$ symmetry group, the chemical potential is in competition with the pion mass for the vacuum structure. It is crucial that this competition be allowed to take place in the low

\footnotetext{
${ }^{2}$ We adopt the convention of Ref. [31] where the diquarks are chosen to have baryon charge 1.
} 
energy effective Lagrangian. At zero baryon chemical potential and nonzero pion mass $S p\left(2 N_{f}\right)$ is left invariant by the vacuum and the parameterization is given by eqs. (3) and (4). However, if $\mu_{B}$ exceeds $m_{\pi}$, a nonzero diquark condensate is expected to form. The combination of the chiral condensate and the diquark condensate leaves only a $S p\left(N_{f}\right)$ invariance, and consequently one must introduce a parameterization which allows for this additional symmetry breaking. A general parameterization is

$$
U=e^{i \frac{\Pi^{i} X^{i}}{v}} \bar{\Sigma}
$$

where $X_{i}$ are the broken generators with respect to $\bar{\Sigma}$. At this point, we consider only an even number of flavors and following Ref. [21] we introduce

$$
\bar{\Sigma}=E \cos (\varphi)+D \sin (\varphi)
$$

where

$$
D \equiv i\left(\begin{array}{cc}
\mathcal{I} & \mathbf{0} \\
\mathbf{0} & \mathcal{I}
\end{array}\right) \quad \text { with } \quad \mathcal{I} \equiv\left(\begin{array}{cc}
0 & -\mathbf{1} \\
\mathbf{1} & 0
\end{array}\right)
$$

The variational parameter $\varphi$ is determined by minimizing the free energy.

After introducing the chemical potential as discussed above, the most general effective Lagrangian containing only pseudo-Goldstone excitations to second order in the chiral counting is

$$
\begin{aligned}
\mathcal{L}(U) & =v^{2} \operatorname{Tr}\left[\partial_{\nu} U \partial^{\nu} U^{\dagger}\right]-i 4 \mu_{B} v^{2} \operatorname{Tr}\left[B U \partial_{0} U^{\dagger}\right]+2 v^{2} \mu_{B}^{2}\left(\operatorname{Tr}\left[B U B^{\mathrm{T}} U^{\dagger}\right]+\operatorname{Tr}[B B]\right) \\
& +v^{2} m_{\pi}^{2} \operatorname{Tr}\left[\mathcal{M} U+\mathcal{M}^{\dagger} U^{\dagger}\right]
\end{aligned}
$$

The phase diagram and dispersion relations for the pseudo-Goldstone modes were derived in [20,21]. The steps leading to the phase diagram are as follows. First, one extremizes the stationary action

$$
\mathcal{L}(\bar{\Sigma})=2 v^{2} \mu_{B}^{2}\left(\operatorname{Tr}\left[B \bar{\Sigma} B^{\mathrm{T}} \bar{\Sigma}^{\dagger}\right]+\operatorname{Tr}[B B]\right)+v^{2} m_{\pi}^{2} \operatorname{Tr}\left[\mathcal{M} \bar{\Sigma}+\mathcal{M}^{\dagger} \bar{\Sigma}^{\dagger}\right]
$$

with respect to $\varphi$. This leads to the following nonanalytic behavior in $\varphi$ :

$$
\begin{array}{r}
\varphi=0 \quad \text { for } \quad \mu_{B}<m_{\pi} \\
\cos (\varphi)=\frac{m_{\pi}^{2}}{\mu_{B}^{2}} \quad \text { for } \quad \mu_{B} \geq m_{\pi} .
\end{array}
$$

It was shown in Ref. [21 that this vacuum direction does indeed parameterize the global minimum of the static potential $\mathcal{L}(\bar{\Sigma})$. The condensates and densities are simply given by derivatives of the static potential with respect to the appropriate sources. For the reader's convenience, we repeat these expressions along with the baryon density

$$
\langle\bar{\psi} \psi\rangle=2 N_{f} G \cos \varphi, \quad\langle\psi \psi\rangle=2 N_{f} G \sin \varphi, \quad n_{B}=8 N_{f} v^{2} \mu_{B} \sin ^{2} \varphi .
$$

The constant $G$ is the chiral condensate in the chiral limit.

We want to stress that the above analysis assumes that the vector spectrum is heavy as compared to the mass of the pseudo-Goldstone excitations. This assumption, however should not hold for large enough number of flavors. Hence it would be very interesting to see if lattice calculations find deviations for $N_{f} \simeq 8$. We also remark that the Lagrangian in eq. (37) is investigated at tree-level.

\section{THE $\epsilon$ TERMS AT NONZERO CHEMICAL POTENTIAL}

In this section we compute the effect of the chemical potential in the $\epsilon$-terms. For simplicity we neglect the spin-one fields and consider only $N_{f}=2$. 


\section{A. Nonzero baryon chemical potential}

The effect of the baryon chemical potential through the $\epsilon$-terms is obtained by substituting $A_{\nu}$ by $B_{\nu}=\mu_{B} \delta_{\nu, 0} B$ in eq. (20) with $C_{i}=C$, for $i=1, \ldots, 10$ and $C_{11}=0$. Since $B_{\nu}$ only has a temporal component, all terms with more than one $B_{\nu}$ vanish on account of the antisymmetry of the differential forms. The resulting term is

$$
\Gamma[U, B]=i \mu_{B} 10 C \int_{M^{4}} \operatorname{Tr}\left[B \alpha^{3}\right]=i \mu_{B} 10 C \int_{M^{4}} \operatorname{Tr}\left[B \alpha_{i} \alpha_{j} \alpha_{k}\right] \epsilon^{0 i j k} d^{4} x .
$$

In general, this term does not vanish and it is instructive to investigate it in more detail. By expanding $\alpha$ to first order in derivatives and lowest order in Goldstone fields, we have the Lagrangian density

$$
i \mu_{B} 10 C \operatorname{Tr}\left[B \alpha_{i} \alpha_{j} \alpha_{k}\right] \epsilon^{0 i j k}=5 \frac{C \mu_{B}}{v^{3}} \epsilon^{0 i j k} \operatorname{Tr}\left[B\left[X^{l}, X^{m}\right] X^{n}\right] \partial_{i} \pi^{l} \partial_{j} \pi^{m} \partial_{k} \pi^{n}+\cdots .
$$

For $N_{f}=2$ the trace is nonzero only if $l, m, n=1,2,3$, that is only the pion generators, $X^{i}$ with $i=1,2,3$ contribute to the trace (see the Appendix for the conventions). An explicit calculation yields

$$
i \mu_{B} 10 C \int_{M^{4}} \operatorname{Tr}\left[B \alpha^{3}\right]=i 5 \frac{C \mu_{B}}{4 \sqrt{2} v^{3}} \int_{M^{4}} \epsilon^{l m n} \epsilon^{0 i j k} \partial_{i} \pi^{l} \partial_{j} \pi^{m} \partial_{k} \pi^{n} d^{4} x+\cdots
$$

The integral is related to the winding number when considering nontrivial topological sectors of the theory and it is naturally coupled to $\mu_{B}$.

In the evaluation of eq. (42), we used the explicit representation of the generators given in eq. (A1). As the diquark condensation sets in and the minimum of the stationary Lagrangian rotates according to eq. (35), this representation breaks down. If, however, we choose to write the rotation of the generators explicitly then we may use the original representation of the generators at the cost of rotating the sources [21]:

$$
B \rightarrow B \cos \varphi-B D E \sin \varphi
$$

For $N_{f}=2$ one can verify that

$$
\epsilon^{0 i j k} \operatorname{Tr}\left[B D E X^{l} X^{m} X^{n}\right] \partial_{i} \pi^{l} \partial_{j} \pi^{m} \partial_{k} \pi^{n}=0,
$$

and the final result is

$$
i \mu_{B} 10 C \int_{M^{4}} \operatorname{Tr}\left[B \alpha^{3}\right]=i 5 \cos (\varphi) \frac{C \mu_{B}}{4 \sqrt{2} v^{3}} \sum_{l, m, n=1}^{3} \int_{M^{4}} \epsilon^{l m n} \epsilon^{0 i j k} \partial_{i} \pi^{l} \partial_{j} \pi^{m} \partial_{k} \pi^{n} d^{4} x+\cdots
$$

where the sum over $l, m, n$ is performed for $l, m, n=1,2,3$. Note that the contribution from the winding number to the baryon density is proportional to $\cos (\varphi)$ and hence decreases with increasing $\mu_{B}$ like $m_{\pi}^{2} / \mu_{B}$.

\section{B. Nonzero isospin chemical potential}

Above we observed that the $\epsilon$ terms couple to the baryon chemical potential via the pions transforming according to the adjoint representation of $S U_{V}\left(N_{f}\right)$. The diquark sector, however, was not active. The situation is reversed when considering a nonzero isospin chemical potential. Since the quarks carry different flavor quantum numbers, it is possible and even natural to introduce different 
chemical potentials for the different flavors. Let us consider $N_{f}=2$ and introduce the chemical potentials

$$
\mu_{B} \equiv \mu_{u}+\mu_{d} \quad \text { and } \quad \mu_{I} \equiv \mu_{u}-\mu_{d}
$$

Here we consider $\mu_{B}=0$. The effects of $\mu_{I}$ in the $\epsilon$ terms enters through the substitution in eq. (20) of $A_{\nu}$ by

$$
I_{\nu} \equiv \mu_{I} \delta_{0 \nu} I \equiv \mu_{I} \delta_{0 \nu} \frac{1}{2}\left(\begin{array}{cc}
\tau^{3} & \mathbf{0} \\
\mathbf{0} & -\tau^{3}
\end{array}\right)
$$

The microscopic two-color Lagrangian is invariant under the combined exchanges $\mu_{I} \leftrightarrow \mu_{B}$ and $d_{L} \leftrightarrow i \sigma_{2} \tau_{2} d_{R}^{*}$ where $\sigma_{2}$ and $\tau_{2}$ are Pauli matrices acting in spin and color space respectively. $\left(d_{L}\right.$ is the left down-quark field and the combination $i \sigma_{2} \tau_{2} d_{R}^{*}$ is known as the conjugate quark state.) This invariance is inherited by the effective Lagrangian where it translates into $\mu_{I} \leftrightarrow \mu_{B}$ and $\left(\pi_{1} X_{1}, \pi_{2} X_{2}\right) \leftrightarrow\left(\pi_{4} X_{4}, \pi_{5} X_{5}\right)$. Using this we conclude from the explicit calculation in the case of baryon chemical potential that for $N_{f}=2$ and $\mu_{B}=0$

$$
i \mu_{I} 10 C \int_{M^{4}} \operatorname{Tr}\left[I \alpha^{3}\right]=i 5 \cos (\varphi) \frac{C \mu_{I}}{4 \sqrt{2} v^{3}} \sum_{l, m, n=3}^{5} \int_{M^{4}} \epsilon^{l m n} \epsilon^{0 i j k} \partial_{i} \pi^{l} \partial_{j} \pi^{m} \partial_{k} \pi^{n} d^{4} x+\ldots,
$$

where now the sum over the flavor indices is performed for $l, m, n=3,4,5$, while $\cos \varphi=\left(m_{\pi} / \mu_{I}\right)^{2}$ for $\mu_{I}>m_{\pi}$ and $\varphi=0$ otherwise. As above, this term is also related to the winding number of the diquark Goldstone fields. We note that in these $\epsilon$-terms $\mu_{B}$ couples only to the isospin triplet $\pi^{1}, \pi^{2}, \pi^{3}$, while $\mu_{I}$ couples only to the baryon triplet $\pi^{3}, \pi^{4}, \pi^{5}$. This should be contrasted with three-color QCD in which the baryon chemical potential couples to the isospin triplet, but there is no coupling at all between the isospin chemical potential and the baryonic sector of the theory. For this reason, the spectrum of solitons in two-color QCD is richer than for $N_{c}=3$. To our knowledge this exciting possibility has not been explored. Note that it is straightforward to extend these results into the $\left(\mu_{B}, \mu_{I}\right)$-plane, using the results of [31]. As a direct application of eq. (42), one can also extend these results to higher $N_{f}$.

Finally we recall that a relevant feature of the Wess-Zumino term is that is required to saturate at the effective Lagrangian level the 't Hooft global anomaly conditions. It is also well known that the Goldstone bosons are sufficient (when chiral symmetry is spontaneously broken) to saturate the anomaly matching conditions. So no other light degree of freedom close to the conformal phase transition is expected to affect the Wess-Zumino term. In the conformal region the underlying quarks will automatically saturate the 't Hooft anomaly conditions. So it would certainly be interesting to monitor these terms close to the conformal phase transition.

\section{THE VECTOR SPECTRUM AT NONZERO CHEMICAL POTENTIAL}

We now examine the effects of a nonzero baryon chemical potential on the spectrum of vectors. The chemical potential enters simply by modifying the derivatives acting on the vector fields:

$$
\partial_{\nu} A_{\rho} \rightarrow \partial_{\nu} A_{\rho}-i\left[B_{\nu}, A_{\rho}\right]
$$

with $B_{\nu}=\mu_{B} \delta_{\nu 0} B \equiv V_{\nu} B$ where $V=\left(\mu_{B}, \overrightarrow{0}\right)$. Using the previous prescription for the vector kinetic term, we find

$$
\operatorname{Tr}\left[F_{\rho \nu} F^{\rho \nu}\right] \rightarrow \operatorname{Tr}\left[F_{\rho \nu} F^{\rho \nu}\right]-4 i \operatorname{Tr}\left[F_{\rho \nu}\left[B^{\rho}, A^{\nu}\right]\right]-2 \operatorname{Tr}\left[\left[B_{\rho}, A_{\nu}\right]\left[B^{\rho}, A^{\nu}\right]-\left[B_{\rho}, A_{\nu}\right]\left[B^{\nu}, A^{\rho}\right]\right] .
$$

Retaining only the quadratic terms in eq. (15a) and integrating by parts yields 


$$
\begin{aligned}
\mathcal{L}_{\text {quad }} & =\frac{1}{2 g^{2}} A_{\rho}^{a}\left\{\delta_{a b}\left[\eta^{\rho \nu} \square-\partial^{\rho} \partial^{\nu}\right]-4 i \gamma_{a b}\left[\eta^{\rho \nu} V \cdot \partial-\frac{1}{2}\left(V^{\rho} \partial^{\nu}+V^{\nu} \partial^{\rho}\right)\right]\right. \\
& \left.+2 \chi_{a b}\left[V \cdot V \eta^{\rho \nu}-V^{\rho} V^{\nu}\right]\right\} A_{\nu}^{b}
\end{aligned}
$$

with

$$
\gamma_{a b}=\operatorname{Tr}\left[T^{a}\left[B, T^{b}\right]\right], \quad \chi_{a b}=\operatorname{Tr}\left[\left[B, T^{a}\right]\left[B, T^{b}\right]\right] .
$$

Note that the inclusion of a baryon chemical potential induces a "magnetic-type" mass term for the vectors at tree-level. To complete the quadratic terms, we include the ordinary mass term already present in eq. (13):

$$
\mathcal{L}_{\text {mass }}=\frac{v^{2}}{2} A_{\rho}^{a} \eta^{\rho \nu} \xi_{a b} A_{\nu}^{b}
$$

with

$$
\xi_{a b}=\frac{1}{2 v^{2} g^{2}}\left\{\left(M_{S}^{2}+M_{X}^{2}\right) \delta_{a b}+2\left(M_{X}^{2}-M_{S}^{2}\right) \operatorname{Tr}\left[T^{a} \bar{\Sigma} T^{b^{T}} \bar{\Sigma}^{\dagger}\right]\right\} .
$$

This term gives the lowest order interaction between the Goldstone sector and the vectors. We assume that the vacuum alignment is fixed by the Goldstone sector and so this interaction term gives the leading order effect of the alignment on the dispersion relations for the vectors 3 .

Having extracted the quadratic terms for the vectors, we can calculate the mass gap, i.e. the zero momentum limit of the dispersion relations. In this limit, the temporal components have no energy dependence while the quadratic part of the spatial components has the form

$$
A_{i}^{a}\left[\delta_{a b} E^{2}-4 \gamma_{a b} \mu_{B} E-g^{2} v^{2} \xi_{a b}-2 \mu_{B}^{2} \chi_{a b}\right] A_{i}^{b} .
$$

Up until this point $N_{f}$ is completely general and the masses of the $\left(2 N_{f}\right)^{2}-1$ vector modes can be obtained by solving for $E$

$$
\operatorname{det}\left[\delta_{a b} E^{2}-4 \gamma_{a b} \mu_{B} E-g^{2} v^{2} \xi_{a b}-2 \mu_{B}^{2} \chi_{a b}\right]=0 .
$$

Here we choose to focous on $N_{f}=2$. The basis of the 15 vectors is taken as that given in the Appendix. We choose the following ordering, $A_{i}^{a}=A_{i}^{a} S^{a}$ for $a=1, \ldots, 10, A_{i}^{a}=A_{i}^{a} X^{a-10}$ for $a=11, \ldots, 15$. Explicitly calculating the traces in (58) using the said basis one finds that the zero momentum propagator matrix is block diagonal with three $1 \times 1$-terms and four $3 \times 3$ blocks. The diagonal terms are for $A_{i}^{1}, A_{i}^{2}$, and $A_{i}^{3}$. The masses in these channels take the single value

$$
M^{a}=M_{S} \quad, \quad a=1,2,3 .
$$

The $3 \times 3$ blocks mix $\left(A_{i}^{5}, A_{i}^{6}, A_{i}^{12}\right),\left(A_{i}^{7}, A_{i}^{8}, A_{i}^{11}\right),\left(A_{i}^{9}, A_{i}^{10}, A_{i}^{13}\right)$, and $\left(A_{i}^{14}, A_{i}^{15}, A_{i}^{4}\right)$ respectively. The first three triplets are degenerate and the masses are obtained by solving for $E$

$$
\left|\begin{array}{ccc}
E^{2}+\mu^{2}-M_{S}^{2} & 2 i E \mu & 0 \\
-2 i E \mu & E^{2}+\mu^{2}-\frac{M_{X}^{2}+M_{S}^{2}}{2}+\frac{M_{X}^{2}-M_{S}^{2}}{2} \cos (2 \varphi) & \frac{M_{X}^{2}-M_{S}^{2}}{2} \sin (2 \varphi) \\
0 & \frac{M_{X}^{2}-M_{S}^{2}}{2} \sin (2 \varphi) & E^{2}-\frac{M_{X}^{2}+M_{S}^{2}}{2}-\frac{M_{X}^{2}-M_{S}^{2}}{2} \cos (2 \varphi)
\end{array}\right|=0
$$

\footnotetext{
${ }^{3}$ We remind the reader that at the tree level there is also a quadratic term of the type $\partial^{\mu} \Pi^{a} A_{\mu}^{a}$. This mixing term can be diagonalized [24.37] away by the field redefinition $A \rightarrow A+v \frac{4-s}{2 M_{A}^{2}} \partial \Pi$ while leaving the mass spectrum unchanged. $A$ stands for the vectors which mix with the pions while $M_{A}^{2}$ is its tree level mass.
} 
while in the $\left(A_{i}^{14}, A_{i}^{15}, A_{i}^{4}\right)$ sector we must solve

$$
\left|\begin{array}{ccc}
E^{2}-\frac{M_{X}^{2}+M_{S}^{2}}{2}+\frac{M_{X}^{2}-M_{S}^{2}}{2} \cos (2 \varphi) & 0 & \frac{M_{X}^{2}-M_{S}^{2}}{2} \sin (2 \varphi) \\
0 & E^{2}+\mu^{2}-\frac{M_{X}^{2}+M_{S}^{2}}{2}-\frac{M_{X}^{2}-M_{S}^{2}}{2} \cos (2 \varphi) & 2 i E \mu \\
\frac{M_{X}^{2}-M_{S}^{2}}{2} \sin (2 \varphi) & -2 i E \mu & E^{2}+\mu^{2}-M_{X}^{2}
\end{array}\right|=0 \text {. }
$$

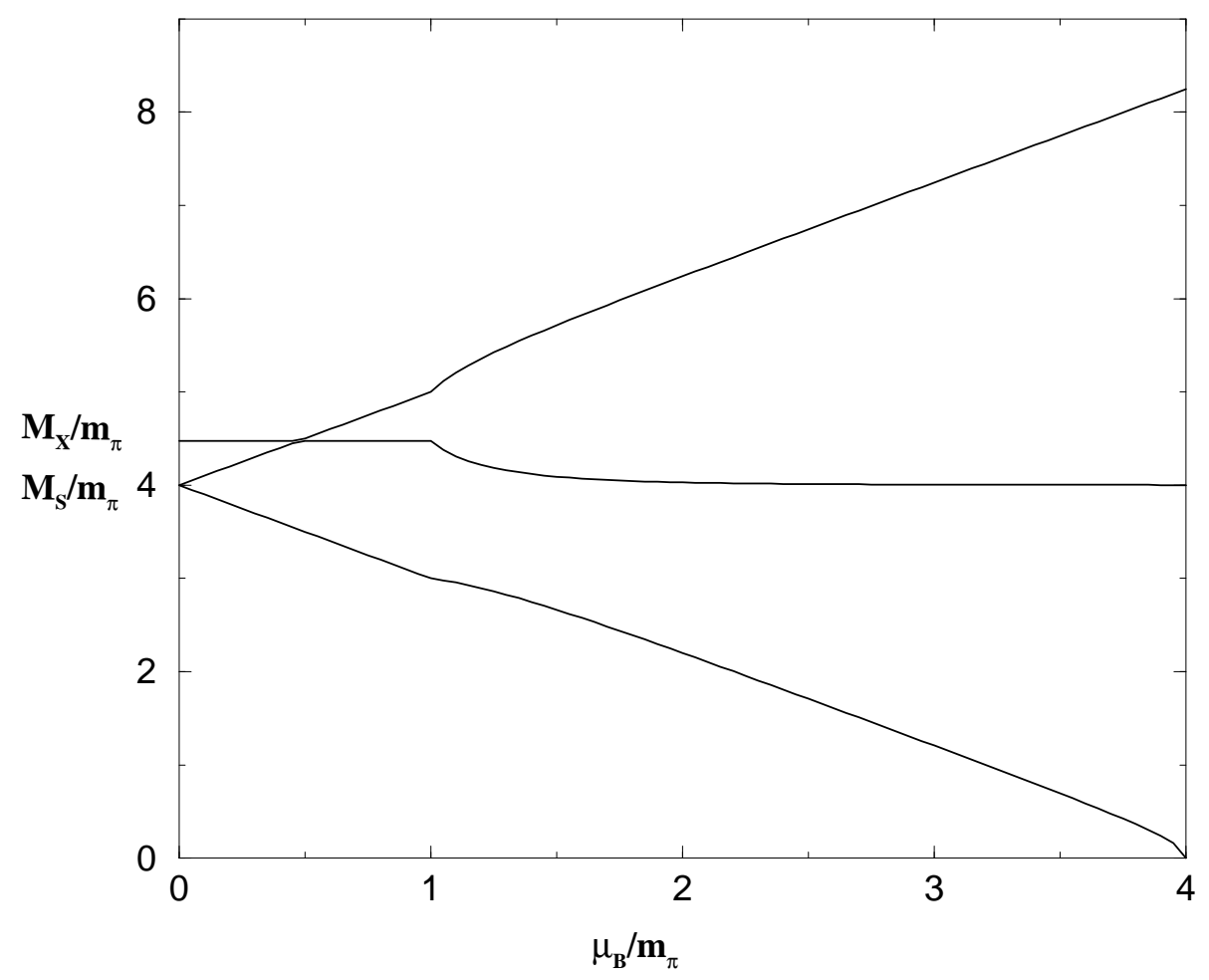

FIG. 2. The triply degenerate masses of the vectors in the sectors $\left(A_{i}^{5}, A_{i}^{6}, A_{i}^{12}\right),\left(A_{i}^{7}, A_{i}^{8}, A_{i}^{11}\right)$, and $\left(A_{i}^{9}, A_{i}^{10}, A_{i}^{13}\right)$. We have choosen $h=s=0, m_{V}=4 m_{\pi} v=m_{\pi}$ and $g=1$ to make the plot. The choice $h=s=0$ realizes the limit where the vector Lagrangian only breaks local chiral symmetry through the term proportional to $m_{V}^{2}$. 


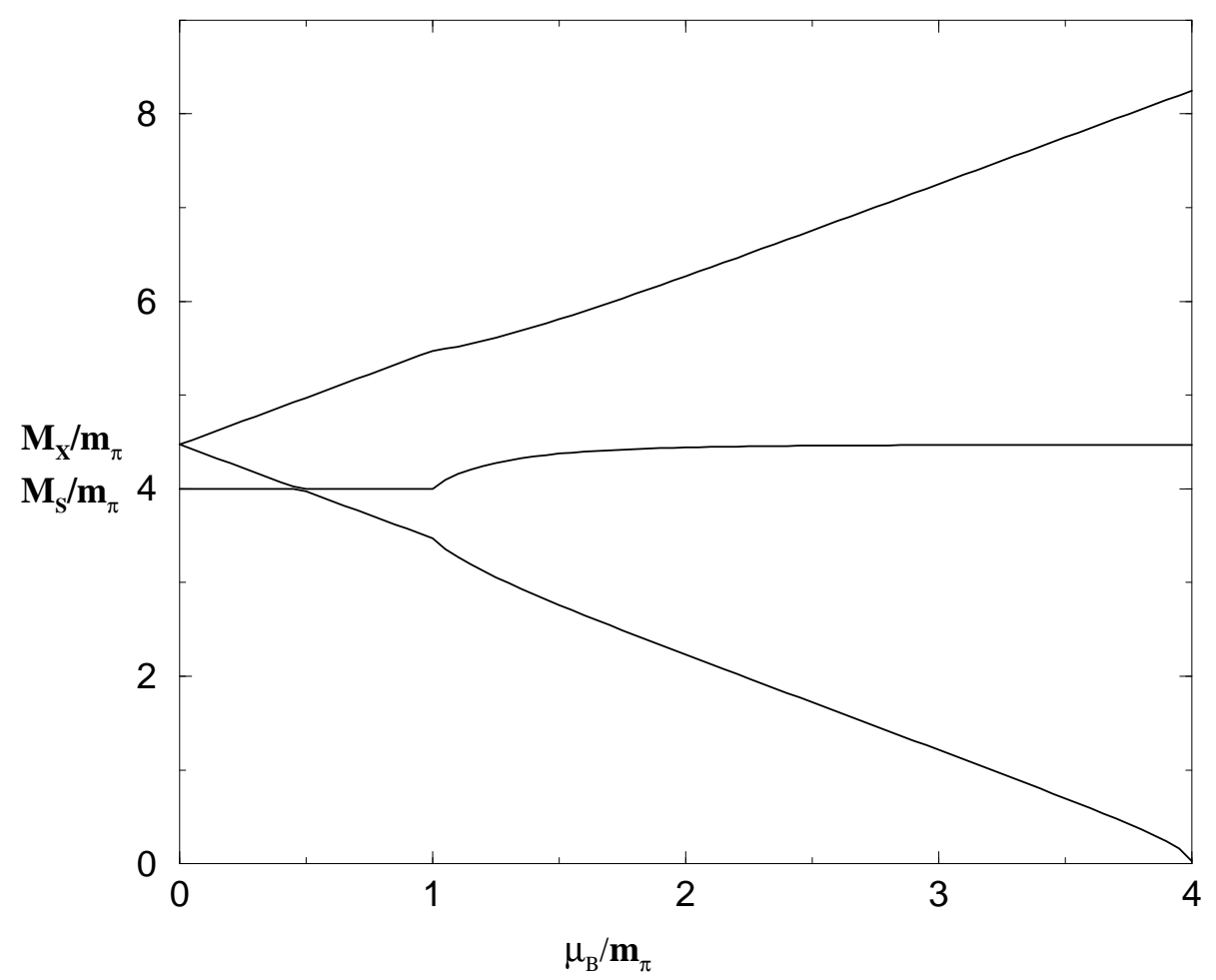

FIG. 3. The masses of the three vectors in the sector $\left(A_{i}^{14}, A_{i}^{15}, A_{i}^{4}\right)$ with the same choice of the parameters $h, s, m_{V}, v, g$ as in figure 2. Note the square-root singularity of the lightest vector mass just below the critical chemical potential in accordence with eq.(67).

To illustrate the calculation of the general zero momentum propagator matrix, we now investigate the "X-type" diquark vector states, i.e. the fields $A_{i}^{14}$ and $A_{i}^{15}$. The $\gamma_{a b}$ and the $\xi_{a b}$ terms do not mix $A_{i}^{14}$ and $A_{i}^{15}$ with the rest of the $15 A_{i}^{a}$ fields. However, the $A_{\nu}^{14}$ and $A_{\nu}^{15}$ states mix since $\left[B, X^{4}\right]=-i X^{5}$ and $\left[B, X^{5}\right]=+i X^{4}$ and so

$$
\gamma_{14,15}=-\gamma_{15,14}=\frac{i}{2}, \quad \chi_{a b}=-\frac{\delta_{a b}}{2}
$$

with $a, b=14,15$. Additionally, the last term in eq. (56) depends on $\bar{\Sigma}$ and it mixes $A_{i}^{14}$ and $A_{i}^{15}$ with $A_{i}^{4}$. This mixing, however, vanishes in two cases:

(i) the nonsuperfluid phase, i.e. where $\mu_{B}<m_{\pi}$ with $\varphi=0$ and $\bar{\Sigma}=E$,

(ii) the superfluid phase with $\mu_{B} \gg m_{\pi}$ where $\varphi \approx \pi / 2$ and $\bar{\Sigma} \approx D$.

In case (i), we find

$$
\xi_{a b}=\frac{M_{X}^{2}}{v^{2}} \delta_{a b} \quad \varphi=0,
$$

with $a, b=4,5$, while for case (ii) we have

$$
\begin{array}{ll}
\xi_{14,14}=\frac{M_{S}^{2}}{v^{2}} & \varphi=\frac{\pi}{2}, \\
\xi_{15,15}=\frac{M_{X}^{2}}{v^{2}} & \varphi=\frac{\pi}{2} .
\end{array}
$$

After diagonalizing the quadratic mass terms, the mass gap in case (i) is 


$$
M_{V^{ \pm}}=M_{X} \pm \mu_{B}
$$

Here $V^{ \pm}$labels the states which diagonalize the mass matrix. In case (ii), we find that

$$
M_{V^{ \pm}}^{2}=\frac{1}{2}\left[M_{S}^{2}+M_{X}^{2}+2 \mu_{B}^{2} \pm \sqrt{\left(M_{S}^{2}-M_{X}^{2}\right)^{2}+8 \mu_{B}^{2}\left(M_{S}^{2}+M_{X}^{2}\right)}\right],
$$

at $\varphi=\pi / 2$. For $\mu_{B}=M_{S}$ or $M_{X}, V^{-}$becomes massless. This suggests that vectors condense for $\mu_{B}=\min \left\{M_{S}, M_{X}\right\}$ breaking rotational invariance. At this value of $\mu_{B}$, the approach breaks down since the effects of such a condensation is not accounted for in eq. (35). This calculation is in agreement with the general solutions plotted in figure 2 and in 3. In these plots we have chosen $h=s=0, m_{V}=4 m_{\pi} v=m_{\pi}$ and $g=1$. This choice leads to the conventional vector mass splitting between the "X"-like and the "S"-like vectors at zero chemical potential. As the triplets consist of two "S"-like and one "X"-like generator or of two "X"-like and one "S"-like generator this mass splitting is apparent on the plots. The "X"-like and "S"-like vectors only mix for $0<\varphi<\pi / 2$, that is only for $\mu_{B}>m_{\pi}$ and $\mu_{B} \gg m_{\pi}$.

The mass gaps of the vectors and the possibility of vector condensation can be studied in lattice simulations. Such studies would give direct information about the conformal phase transition since the masses of the vectors depend directly on the parameters $s, h$, and $m_{V}$. Fixing these parameters is sufficient to check our conjecture that for a small number of light flavors we can use an approximate local chiral gauge theory to introduce the vector-Goldstone interactions (i.e. $h=s=0$ but $m_{V}^{2} \neq 0$ ), while for a large number of flavors an enhanced global symmetry (i.e. $s=2 h=4$ ) may emerge. We hope that lattice simulations can shed light on this issue in the near future.

\section{THE TWO-COLOR LINEAR EFFECTIVE LAGRANGIAN}

In this section, we study the effective Lagrangian for two-color QCD for which the chiral symmetry is linearly realized. The authors of Ref. [42] used this theory to study the superfluid phase transition at nonzero baryon chemical potential and zero isospin chemical potential. This theory was also used in Ref. [13] to examine the universal properties of the chiral symmetry restoring phase transition of two-color QCD at nonzero temperature and vanishing chemical potential. The random matrix model for two-color QCD at nonzero chemical potential considered by the authors of Ref. [44] also bears resemblence to the linear sigma model considered here. We begin by introducing the effective Lagrangian including the couplings between the pseudo-Goldstone excitations and the spinone sector. We then derive the phase diagram at nonzero baryon and isospin chemical potential.

In the linear effective Lagrangian, the $2 N_{f}^{2}-N_{f}-1$ Goldstone fields which are present in the nonlinear effective Lagrangian are accompanied by a scalar particle, $\sigma$. These fields are elements of the antisymmetric $2 N_{f} \times 2 N_{f}$ matrix

$$
M=\frac{1}{2 \sqrt{2}}\left(\sigma-i 2 \sqrt{2} \pi^{a} X^{a}\right) E .
$$

Under the action of $u \in \mathrm{SU}\left(2 N_{f}\right), M$ transforms as

$$
M \rightarrow u M u^{T} .
$$

A vector field, $A_{\nu}$, can be introduced in a fashion similar to that for the nonlinear effective Lagrangian and so transforms according to eq. (10). Hence, it is useful to define the covariant derivative acting on $M$

$$
D_{\nu} M=\partial_{\nu} M-i A_{\nu} M-i M A_{\nu}^{T} .
$$

As in the nonlinear realization, we write a general Lagrangian consistent with the global chiral symmetry invariance: 


$$
\begin{aligned}
\mathcal{L}_{\text {linear }} & =\operatorname{Tr}\left[\partial_{\nu} M \partial^{\nu} M^{\dagger}\right]-m^{2} \operatorname{Tr}\left[M M^{\dagger}\right]+i c_{1} \operatorname{Tr}\left[A_{\nu}\left(M \partial^{\nu} M^{\dagger}-\partial^{\nu} M M^{\dagger}\right)\right] \\
& +c_{2} \operatorname{Tr}\left[A^{\nu} M A_{\nu}^{T} M^{\dagger}\right]+c_{3} \operatorname{Tr}\left[A_{\nu} A^{\nu}\right]+c_{4} \operatorname{Tr}\left[A_{\nu} A^{\nu} M M^{\dagger}\right] \\
& -\lambda_{1} \operatorname{Tr}\left[M M^{\dagger}\right]^{2}-\lambda_{2}\left(\operatorname{Tr}\left[M M^{\dagger}\right]\right)^{2}-H \operatorname{Re}(\operatorname{Tr}[\mathcal{M} M]) / \sqrt{2} \\
& -\frac{1}{2 g^{2}} \operatorname{Tr}\left[F_{\mu \nu} F^{\mu \nu}\right]
\end{aligned}
$$

where $\mathcal{M}$ is given by eq. (68), $F_{\mu \nu}$ is given in eq. (15b) and all the coefficients are real. Since it is determined by the same symmetry principle, the linear effective Lagrangian is very similar in form to the nonlinear version. We have only included potential terms up to fourth order in the mass dimension. The Gell-Mann-Oakes-Renner relation fixes the coefficient of the explicit symmetry breaking term to be $H=m_{\pi}^{2} f_{\pi}$, where $m_{\pi}$ is the degenerate mass of the pseudo-Goldstone particles at zero baryon and isospin chemical potential and $f_{\pi}=2 \sqrt{2} v$. For simplicity, we are ignoring terms which account for the explicit breaking of the anomalous $U(1)_{A}$ symmetry group.

For the purposes of deriving the phase diagram, we consider only $N_{f}=2$ in the absence of spinone particles. As discussed above, the chemical potentials can be introduced by formally replacing the usual derivatives with the covariant derivative

$$
\partial_{\nu} M \rightarrow \partial_{\nu} M-i\left(\mu_{B} B+\mu_{I} I\right) \delta_{0 \nu} M-i M\left(\mu_{B} B+\mu_{I} I\right) \delta_{0 \nu},
$$

where

$$
\mu_{B} B+\mu_{I} I=\frac{\mu_{B}}{2}\left(\begin{array}{cc}
\mathbf{1} & \mathbf{0} \\
\mathbf{0} & \mathbf{- 1}
\end{array}\right)+\frac{\mu_{I}}{2}\left(\begin{array}{cc}
\tau^{3} & \mathbf{0} \\
\mathbf{0} & -\tau^{3}
\end{array}\right) .
$$

The linear effective Lagrangian at nonzero baryon and isospin chemical potential is then

$$
\begin{aligned}
\mathcal{L}_{\text {linear }} & =\operatorname{Tr}\left[\partial_{\nu} M \partial^{\nu} M^{\dagger}\right]-m^{2} \operatorname{Tr}\left[M M^{\dagger}\right]-4 \lambda_{1} \operatorname{Tr}\left[M M^{\dagger}\right]^{2}-4 \lambda_{2}\left(\operatorname{Tr}\left[M M^{\dagger}\right]\right)^{2} \\
& +2 \operatorname{Tr}\left[M\left(\mu_{B} B+\mu_{I} I\right) M^{\dagger}\left(\mu_{B} B+\mu_{I} I\right)+M M^{\dagger}\left(\mu_{B} B+\mu_{I} I\right)^{2}\right] \\
& +4 i \operatorname{Tr}\left[\left(\mu_{B} B+\mu_{I} I\right) \partial_{0} M M^{\dagger}\right]-\frac{H}{\sqrt{2}} \operatorname{Re}(\operatorname{Tr}[\mathcal{M} M]) .
\end{aligned}
$$

For $N_{f}=2$, there is a triplet of pions, a scalar quark-antiquark bound state, a scalar diquark, and its antiparticle. The diquarks carry baryon charge \pm 1 and the triplet of pions have isospin charges \pm 1 and 0 . Computing the traces in $\mathcal{L}$ for $N_{f}=2$, one finds the effective potential

$$
V=\frac{m^{2}}{2}\left(\sigma^{2}+\pi^{a} \pi^{a}\right)+\frac{\lambda}{4}\left(\sigma^{2}+\pi^{a} \pi^{a}\right)^{2}-\frac{\mu_{I}^{2}}{2}\left[\left(\pi^{1}\right)^{2}+\left(\pi^{2}\right)^{2}\right]-\frac{\mu_{B}^{2}}{2}\left[\left(\pi^{4}\right)^{2}+\left(\pi^{5}\right)^{2}\right]-H \sigma .
$$

There is no distinction between the two quartic terms in eq. (74) for $N_{f}=2$, and so the two couplings, $\lambda_{1}$ and $\lambda_{2}$, have been absorbed into a single coupling, $\lambda$. The coefficients $m$ and $\lambda$ may be expressed in terms of $m_{\pi}, m_{\sigma}$ and $f_{\pi}$ :

$$
\begin{gathered}
m^{2}=\frac{3 m_{\pi}^{2}-m_{\sigma}^{2}}{2} \\
\lambda=\frac{m_{\sigma}^{2}-m_{\pi}^{2}}{2 f_{\pi}^{2}} .
\end{gathered}
$$

The potential is manifestly invariant under a $O(2) \times O(2)$ symmetry group, i.e. under independent rotations of $\left(\pi^{1}, \pi^{2}\right)$ and $\left(\pi^{4}, \pi^{5}\right)$. An explicit calculation shows that at the level of the Lagrangian this invariance is not violated by the term linear in the time derivative:

$$
4 i \operatorname{Tr}\left[\left(\mu_{B} B+\mu_{I} I\right) \partial_{0} M M^{\dagger}\right]=\mu_{I}\left(\pi^{1} \partial_{0} \pi^{2}-\pi^{2} \partial_{0} \pi^{1}\right)+\mu_{B}\left(\pi^{5} \partial_{0} \pi^{4}-\pi^{4} \partial_{0} \pi^{5}\right) .
$$


We denote the pion condensate by $\langle\pi\rangle$, the diquark condensate by $\langle D\rangle$ and the chiral condensate by $\langle\sigma\rangle$. Since $\pi^{3}$ does not couple to either chemical potential, it is not expected to condense. The $O(2) \times O(2)$ symmetry invariance of the effective potential requires that

$$
\left\langle\pi^{1}\right\rangle^{2}+\left\langle\pi^{2}\right\rangle^{2}=\langle\pi\rangle^{2}, \quad\left\langle\pi^{4}\right\rangle^{2}+\left\langle\pi^{5}\right\rangle^{2}=\langle D\rangle^{2},
$$

in the unbroken phase. The values of the condensates as a function of the baryon and isospin chemical potentials are found by extremizing the effective potential:

$$
\begin{aligned}
& \frac{\delta V}{\delta\langle\sigma\rangle}=0=-H+\langle\sigma\rangle\left[m^{2}+\lambda\left(\langle\sigma\rangle^{2}+\langle\pi\rangle^{2}+\langle D\rangle^{2}\right)\right] \\
& \frac{\delta V}{\delta\langle\pi\rangle}=0=\langle\pi\rangle\left[m^{2}-\mu_{I}^{2}+\lambda\left(\langle\sigma\rangle^{2}+\langle\pi\rangle^{2}+\langle D\rangle^{2}\right)\right] \\
& \frac{\delta V}{\delta\langle D\rangle}=0=\langle D\rangle\left[m^{2}-\mu_{B}^{2}+\lambda\left(\langle\sigma\rangle^{2}+\langle\pi\rangle^{2}+\langle D\rangle^{2}\right)\right] .
\end{aligned}
$$

There are three real solutions to this set of equations corresponding to three distinct phases. The first solution is given by $\langle\pi\rangle=\langle D\rangle=0$ with $\langle\sigma\rangle$ determined by the nonzero solution to $H=m_{\pi}^{2} f_{\pi}=$ $\langle\sigma\rangle\left(m^{2}+\lambda\langle\sigma\rangle\right)$. In this phase, $\langle\sigma\rangle$ is constant, i.e. equal to $f_{\pi}$. The other two solutions are

$$
\begin{aligned}
\langle\sigma\rangle & =\frac{H}{\mu_{B}^{2}} \\
\langle D\rangle & =\sqrt{\frac{\mu_{B}^{2}-m^{2}-\frac{\lambda H^{2}}{\mu_{B}^{4}}}{\lambda}} \\
\langle\pi\rangle & =0,
\end{aligned}
$$

and

$$
\begin{aligned}
\langle\sigma\rangle & =\frac{H}{\mu_{I}^{2}} \\
\langle D\rangle & =0 \\
\langle\pi\rangle & =\sqrt{\frac{\mu_{I}^{2}-m^{2}-\frac{\lambda H^{2}}{\mu_{I}^{4}}}{\lambda} .}
\end{aligned}
$$

Note that even for nonzero quark masses, $\langle\pi\rangle$ and $\langle D\rangle$ are true order parameters. The phase boundaries in the $\left(\mu_{I}, \mu_{B}\right)$ plane can now be calculated and are given by solving the inequalities

$$
\frac{\delta^{2} V}{\delta\langle D\rangle \delta\langle D\rangle}(\langle\sigma\rangle,\langle\pi\rangle, 0)>0
$$

and

$$
\frac{\delta^{2} V}{\delta\langle\pi\rangle \delta\langle\pi\rangle}(\langle\sigma\rangle, 0,\langle D\rangle)>0
$$

For $\mu_{B}<\mu_{I}$, the inequalities are satisfied for $\mu_{I}<m_{\pi}$, and for $\mu_{B}>\mu_{I}, \mu_{B}<m_{\pi}$. One may check that the second derivatives of the potential vanish on these critical lines indicating that the transition is of second order. Along the line $\mu_{B}=\mu_{I}>m_{\pi}$, the transition is first-order since the baryon and isospin densities are discontinuous across this line. This is exactly the phase diagram which is predicted by chiral perturbation theory [31].

The dispersion relations can now be calculated by examining the quadratic terms in eq. (74). The Lorentz breaking term in eq. (74) induces a mixing in the $\left(\pi^{1}, \pi^{2}\right)$ and $\left(\pi^{4}, \pi^{5}\right)$ sectors. For the two unmixed states, the dispersion relations are 


$$
\begin{array}{rlrl}
\sigma & E & =\sqrt{\mathbf{p}^{2}+m^{2}+\lambda\left(3\langle\sigma\rangle^{2}+\langle\pi\rangle^{2}+\langle D\rangle^{2}\right)} \\
\pi^{3} & E & =\sqrt{\mathbf{p}^{2}+m^{2}+\lambda\left(\langle\sigma\rangle^{2}+\langle\pi\rangle^{2}+\langle D\rangle^{2}\right)} .
\end{array}
$$

The dispersion relations for the $\left(\pi^{1}, \pi^{2}\right)$ sector are obtained by solving

$$
\operatorname{det}\left(\begin{array}{cc}
z_{1} & -2 i \mu_{I} E \\
2 i \mu_{I} E & z_{2}
\end{array}\right)=0 \text {, }
$$

where the diagonal terms are

$$
\begin{aligned}
& z_{1} \equiv \mathbf{p}^{2}-E^{2}+m^{2}+\lambda\left(\langle\sigma\rangle^{2}+3\langle\pi\rangle^{2}+\langle D\rangle^{2}\right)-\mu_{I}^{2} \\
& z_{2} \equiv \mathbf{p}^{2}-E^{2}+m^{2}+\lambda\left(\langle\sigma\rangle^{2}+\langle\pi\rangle^{2}+\langle D\rangle^{2}\right)-\mu_{I}^{2}
\end{aligned}
$$

The dispersion relations for the $\left(\pi^{4}, \pi^{5}\right)$ can be found by exchanging $\mu_{I}$ and $\mu_{B}$ in the dispersion relations for the $\left(\pi^{1}, \pi^{2}\right)$ sector (we denote the propagating modes in the diquark sector by $D_{ \pm}$). The dispersion relations in the nonsuperfluid phase, $\mu_{I}, \mu_{B}<m_{\pi}$, are

$$
\begin{aligned}
E_{\sigma} & =\sqrt{\mathbf{p}^{2}+m_{\pi}^{2}+2 \lambda f_{\pi}^{2}} \\
E_{\pi_{0}} & =\sqrt{\mathbf{p}^{2}+m_{\pi}^{2}} \\
E_{\pi_{ \pm}} & =\sqrt{\mathbf{p}^{2}+m_{\pi}^{2}} \pm \mu_{I} \\
E_{D_{ \pm}} & =\sqrt{\mathbf{p}^{2}+m_{\pi}^{2}} \pm \mu_{B} .
\end{aligned}
$$

In the phase with the condensation of pions, the dispersion relations are

$$
\begin{aligned}
E_{\sigma}^{2} & =\mathbf{p}^{2}+\frac{2 \lambda H^{2}}{\mu_{I}^{4}}+\mu_{I}^{2} \\
E_{\pi_{0}}^{2} & =\mathbf{p}^{2}+\mu_{I}^{2} \\
E_{\pi_{ \pm}}^{2} & =\mathbf{p}^{2}-m^{2}-\frac{\lambda H^{2}}{\mu_{I}^{4}}+3 \mu_{I}^{2} \pm \sqrt{\left(\frac{\lambda H^{2}}{\mu_{I}^{4}}+m^{2}-3 \mu_{I}^{2}\right)^{2}+4 \mu_{I}^{2} \mathbf{p}^{2}} \\
E_{D_{ \pm}}^{2} & =\mathbf{p}^{2}+\mu_{I}^{2}+\mu_{B}^{2} \pm 2 \mu_{B} \sqrt{\mathbf{p}^{2}+\mu_{I}^{2}} .
\end{aligned}
$$

Note that for $\mu_{I}>m_{\pi}$, the $\pi_{+}$is the massless Goldstone boson which arises on account of the spontaneous breaking of baryon number. In the phase with the diquark condensate, the dispersion relations can be obtained by interchanging $\mu_{I}$ with $\mu_{B}$ and $\pi_{ \pm}$with $D_{ \pm}$. In this phase, the $D_{+}$is the Goldstone mode.

The fermionic determinant for two-color QCD is real but not positive at nonzero chemical potential. Therefore, it is only possible to study cases where an equal number of flavors share a given chemical potential. The two-flavor case discussed above is thus only accessible to lattice simulations at either $\mu_{I}=0$ or $\mu_{B}=0$. If one doubles the number of flavors and gives two flavors a chemical potential $\mu_{u}$ and the other two flavors a chemical potential $\mu_{d}$, then the theory has positivity in the full $\left(\mu_{B}, \mu_{I}\right)$-phase. The phase diagram, however, has a different form than the one above [31].

\section{CONCLUSION}

In this work, we have examined the phase structure of two-color QCD as a function of the baryon and isospin chemical potentials as well as the number of light quark flavors via effective Lagrangians. We first considered the case for which the chiral symmetry group is nonlinearly realized. In addition to the pseudo-Goldstone excitations, we augmented the theory with the sector of spin-one particles and the negative intrinsic parity terms for the group $S U\left(2 N_{f}\right)$. 
We reviewed the salient aspects of the conformal phase transition which is expected to occur as the number of light flavors is increased. Since this phase transition strongly affects the phase structure as a function of the chemical potentials, we suggested different ways in which lattice simulations should cast further light on these issues. We demonstrated that new terms in the chemical potentials exist in the negative intrinsic parity sector at the level of the effective Lagrangian. Such terms are expected to play an important role when analyzing the solitonic structure of $N_{c}=2$ QCD. Since the baryons are also Goldstone bosons, the solitonic structure is naturally richer than for $N_{c}=3$ QCD. For example, just as $\mu_{B}$ couples to the winding number of the pion sector, we showed that $\mu_{I}$ couples to the winding number of the diquark sector. Future investigations into this matter will certainly be interesting.

Also, unlike $N_{c}=3 \mathrm{QCD}$, some of the spin-one particles of $N_{c}=2$ QCD are charged under baryon number. We have hence analyzed this sector at nonzero baryon chemical potential. We observed novel features such as the possible condensation of vector particles. We were able to calculate the value of the chemical potential at which the vectors condense and thereby break rotational invariance. Our results provide new avenues for future investigations. We then turned to the linear effective Lagrangian including the spin-one sector. The phase diagram that is predicted by the linear effective Lagrangian matches that predicted by chiral perturbation theory.

Our results can be extended to describe QCD-like theories with quarks in the adjoint representation of the gauge group for an arbitrary number of colors. Another possible area of investigation is standard QCD with a nonzero isospin chemical potential [45]. Both cases are suitable for lattice studies. For example, ordinary QCD at nonzero strange and isospin chemical potentials has been studied in Ref. [46]. The inclusion of the vector mesons may alter the phase structure predicted in Ref. 46. since the average mass splitting between the spin-one sector and the spin-zero is not dramatically large phenomenologically. In fact, the spin-one sector was shown in Ref. [47] to be crucial to the dynamics of the light degrees of freedom.

\section{ACKNOWLEDGMENTS}

The authors thank A. Jackson, R.L. Jaffe, J. Schechter, J. Skullerud, D. Toublan and J. Verbaarschot for useful discussions.

\section{APPENDIX A: EXPLICIT REALIZATION OF THE $S P(4)$ GENERATORS}

The generators of $S U(4)$ can be conveniently represented as

$$
S^{a}=\left(\begin{array}{cc}
\mathbf{A} & \mathbf{B} \\
\mathbf{B}^{\dagger} & -\mathbf{A}^{T}
\end{array}\right), \quad X^{i}=\left(\begin{array}{cc}
\mathbf{C} & \mathbf{D} \\
\mathbf{D}^{\dagger} & \mathbf{C}^{T}
\end{array}\right),
$$

where $A$ is hermitian, $C$ is hermitian and traceless, $B=B^{T}$ and $D=-D^{T}$. The $\{S\}$ are also a representation of the $S p(4)$ generators since they obey the relation $S^{T} E+E S=0$. We define

$$
S^{a}=\frac{1}{2 \sqrt{2}}\left(\begin{array}{cc}
\tau^{a} & \mathbf{0} \\
\mathbf{0} & -\tau^{a T}
\end{array}\right), \quad a=1,2,3,4 .
$$

For $a=1,2,3$, we have the standard Pauli matrices, while for $a=4$ we define $\tau^{4}=\mathbf{1}$. These are simply the generators for $S U_{V}(2) \times U_{V}(1)$. For $a=5, \ldots, 10$

$$
S^{a}=\frac{1}{2 \sqrt{2}}\left(\begin{array}{cc}
\mathbf{0} & \mathbf{B}^{a} \\
\mathbf{B}^{a \dagger} & \mathbf{0}
\end{array}\right), \quad a=5, \ldots, 10
$$

and 


$$
\begin{array}{ccc}
B^{5}=1 & B^{7}=\tau^{3} & B^{9}=\tau^{1} \\
B^{6}=i 1 & B^{8}=i \tau^{3} & B^{10}=i \tau^{1}
\end{array}
$$

The five axial type generators $\left\{X^{i}\right\}$ are

$$
X^{i}=\frac{1}{2 \sqrt{2}}\left(\begin{array}{cc}
\tau^{i} & \mathbf{0} \\
\mathbf{0} & \tau^{i T}
\end{array}\right), \quad i=1,2,3
$$

where $\tau^{i}$ are the standard Pauli matrices. For $i=4,5$

$$
X^{i}=\frac{1}{2 \sqrt{2}}\left(\begin{array}{cc}
\mathbf{0} & \mathbf{D}^{i} \\
\mathbf{D}^{i \dagger} & \mathbf{0}
\end{array}\right), \quad i=4,5,
$$

and

$$
D^{4}=\tau^{2}, \quad D^{5}=i \tau^{2}
$$

The generators are normalized as follows

$$
\operatorname{Tr}\left[S^{a} S^{b}\right]=\operatorname{Tr}\left[X^{a} X^{b}\right]=\frac{1}{2} \delta^{a b}, \quad \operatorname{Tr}\left[X^{i} S^{a}\right]=0 .
$$


[1] For two recent reviews and a rather complete list of references, see K. Rajagopal and F. Wilczek, hep-ph/0011333, and M.G. Alford, hep-ph/0102047.

[2] B. C. Barrois, Nucl. Phys. B 129, 390 (1977).

[3] D. Bailin and A. Love, Phys. Rept. 107, 325 (1984).

[4] R. Ouyed and F. Sannino, astro-ph/0103022. R. Ouyed and F. Sannino, Phys. Lett. B511, 66 (2001), hep-ph/0103168.

[5] D. K. Hong, S. D. Hsu and F. Sannino, Phys. Lett. B 516, 362 (2001), hep-ph/0107017.

[6] M. P. Lombardo, hep-ph/0103141.

[7] R. D. Mawhinney, Nucl. Phys. Proc. Suppl. 60A, 306 (1998), hep-lat/9705030; D. Chen, R. D. Mawhinney, Nucl. Phys. Proc. Suppl. 53, 216 (1997).

[8] S. Morrison and S. Hands, hep-lat/9902012.

[9] S. Hands, J. B. Kogut, M. Lombardo and S. E. Morrison, Nucl. Phys. B 558, 327 (1999), hep-lat/9902034.

[10] R. Aloisio, A. Galante, V. Azcoiti, G. Di Carlo and A. F. Grillo, hep-lat/0007018.

[11] Y. Liu, O. Miyamura, A. Nakamura and T. Takaishi, hep-lat/0009009.

[12] R. Aloisio, V. Azcoiti, G. Di Carlo, A. Galante and A. F. Grillo, Phys. Lett. B 493, 189 (2000), hep-lat/0009034.

[13] E. Bittner, M. Lombardo, H. Markum and R. Pullirsch, Nucl. Phys. Proc. Suppl. 94, 445 (2001), hep-lat/0010018.

[14] S. J. Hands, B. Kogut, S. E. Morrison and D. K. Sinclair, Nucl. Phys. Proc. Suppl. 94, 457 (2001), hep-lat/0010028.

[15] S. Muroya, A. Nakamura and C. Nonaka, Nucl. Phys. Proc. Suppl. 94, 469 (2001), hep-lat/0010073.

[16] S. Hands, I. Montvay, M. Oevers, L. Scorzato and J. Skullerud, Nucl. Phys. Proc. Suppl. 94, 461 (2001), hep-lat/0010085.

[17] R. Aloisio, V. Azcoiti, G. Di Carlo, A. Galante and A. F. Grillo, Nucl. Phys. B 606, 322 (2001), hep-lat/0011079.

[18] J. B. Kogut, D. Toublan and D. K. Sinclair, Phys. Lett. B 514, 77 (2001), hep-lat/0104010.

[19] J. B. Kogut, D. K. Sinclair, S. J. Hands and S. E. Morrison, hep-lat/0105026.

[20] J.B. Kogut, M.A. Stephanov, and D. Toublan, Phys. Lett. B 464 (1999) 183-191.

[21] J.B. Kogut, M.A. Stephanov, D. Toublan, J.J.M. Verbaarschot, and A. Zhitnitsky, Nucl. Phys. B 582 (2000) 477-513.

[22] S. Weinberg, Physica 96A (1979) 327; J. Gasser and H. Leutwyler, Ann. Phys. (N.Y.) 158 (1984) 142; Nucl. Phys. B250 (1985) 465.

[23] K. Intriligator and N. Seiberg, Nucl. Phys. Proc. Suppl. 45BC, 1 (1996); N. Seiberg, Phys. Rev. D49, 6857 (1994); Nucl. Phys. B 435, 129 (1995); F. Sannino and J. Schechter, Phys. Rev. D57, 170 (1998); D.H. Hsu, F. Sannino and J. Schechter, Phys. Lett. B427, 300 (1998).

[24] T. Appelquist, P.S. Rodrigues da Silva and F. Sannino, Phys. Rev. D60, 116007 (1999), hep-ph/9906555.

[25] T. Appelquist and F. Sannino, Phys. Rev. D59, 067702 (1999).

[26] T. Appelquist, J. Terning and L.C.R. Wijewardhana, Phys. Rev. Lett. 77, 1214 (1996); T. Appelquist, Dynamical Electroweak Symmetry Breaking. School of Particles and Fields, Mexico City, 1990; T. Appelquist and S. Selipsky, Phys. Lett. B400, 364 (1997); M. Velkovsky and E. Shuryak, Phys. Lett. B437, 398 (1998).

[27] F. Sannino and J. Schechter, Phys. Rev. D60, 056004 (1999).

[28] Z. Duan, P.S. Rodrigues da Silva and F. Sannino, Nucl. Phys. B 592, 371 (2001), hep-ph/0001303.

[29] J. Wess and B. Zumino, Phys. Lett. B37, 95 (1971).

[30] E. Witten, Nucl. Phys. B223, 422 (1983); Nucl. Phys. B223, 433 (1983).

[31] K. Splittorff, D. T. Son and M. A. Stephanov, Phys. Rev. D 64, 016003 (2001), hep-ph/0012274.

[32] J. Wess and J. Bagger, Supersymmetry and Supergravity, Princeton Univ. Press, Princeton, New Jersey.

[33] M. E. Peskin, Nucl. Phys. B 175 (1980) 197.

[34] T. Appelquist, Z. Duan and F. Sannino, Phys.Rev. D61 (2000) 125009, hep-ph/0001043.

[35] M. Bando, T. Kugo and K. Yamawaki, Phys. Rept. 164, 217 (1988). 
[36] Ö. Kaymakcalan, S. Rajeev and J. Schechter, Phys. Rev. D30, 594 (1984); J. Schechter, Phys. Rev. D34, 868 (1986); P. Jain, R. Johnson, Ulf-G. Meissner, N. W. Park and J. Schechter, Phys. Rev. D37, 3252 (1988).

[37] Ö. Kaymakcalan and J. Schechter, Phys. Rev. D31, 1109 (1985).

[38] R.G. Leigh and M.J. Strassler, Nucl. Phys.B496, 132 (1997).

[39] D. J. Gross and F. Wilczek, Phys. Rev. Lett. 30, 1343 (1973).

[40] H. D. Politzer, Phys. Rev. Lett. 30, 1346 (1973).

[41] K. Splittorff, D. Toublan and J. J. Verbaarschot, hep-ph/0108040.

[42] R. Rapp, T. Schafer, E. V. Shuryak and M. Velkovsky, Phys. Rev. Lett. 81, 53 (1998), hep-ph/9711396.

[43] J. Wirstam, Phys. Rev. D62 (2000) 045012, hep-ph/9912446.

[44] B. Vanderheyden and A.D. Jackson, Phys. Rev. D 64, 074016 (2001), hep-ph/0102064.

[45] D.T. Son and M.A. Stephanov, Phys. Rev. Lett. 86 (2001) 592-595, hep-ph/0005225; Phys. Atom. Nucl. 64, 834 (2001) [Yad. Fiz. 64, 899 (2001)], hep-ph/0011365.

[46] J. B. Kogut and D. Toublan, Phys. Rev. D 64, 034007 (2001), hep-ph/0103271.

[47] M. Harada, F. Sannino and J. Schechter, Phys. Rev. Lett. 78, 1603 (1997), hep-ph/9609428. 